\title{
Ethnomathématique au Brésil et en Europe: un dialogue
}

Eric Vandendriessche $\mathbf{1}$
Centre national de la recherche scientifique $(\mathrm{CNRS})$ et Université de Paris, Laboratoire "Sciences,
Philosophie, Histoire" (SPHere), Paris, France

\author{
Maria Cecilia Fantinato ${ }^{2}$ (D) \\ Universidade Federal Fluminense (UFF), Faculdade de Educação, Niterói, RJ, Brasil
}

\begin{abstract}
Résumé
A l'occasion de ce numéro spécial de REMat consacré à l'ethnomathématique dans ses liens avec la "décolonisation et les mouvements de résistance", l'ethnomathématicienne et didacticienne brésilienne Maria Cecilia Fantinato et l'ethnomathématicien français Éric Vandendriessche ont souhaité contribuer à ce volume par un dialogue sur le thème de l'ethnomathématique. La discussion retranscrite dans cet article aborde ainsi tour à tour certaines questions relatives au développement de ce jeune champ interdisciplinaire dans différents contextes. Dans un premier temps, les deux chercheurs retracent les circonstances de leur première collaboration. La discussion porte ensuite sur le développement de l'ethnomathématique au Brésil et en Europe, ainsi que sur les relations que ce domaine de recherche entretient avec l'anthropologie, l'épistémologie, et les sciences de l'éducation. Enfin, les deux ethnomathématiciens s'interrogent sur les défis actuels de l'ethnomathématique en tant que domaine de recherche international.
\end{abstract}

Mots-clés: Ethnomathématique; Dialogue; Éducation; Brésil; Europe.

\section{Etnomatemática no Brasil e na Europa: um diálogo}

\section{Resumo}

Por ocasião desta edição especial da REMat dedicada à etnomatemática em seus vínculos com a "perspectiva decolonial e os movimentos de resistência", a etnomatemática e educadora brasileira Maria Cecilia Fantinato e o etnomatemático francês Éric Vandendriessche buscaram contribuir para este dossiê com um diálogo sobre o tema da etnomatemática. A discussão transcrita neste artigo aborda assim, por sua vez, algumas questões relativas ao desenvolvimento desse jovem campo interdisciplinar em diferentes contextos. Inicialmente, os dois pesquisadores reconstituem as

Submetido em: 26/06/2021 Aceito em: 18/08/2021 Publicado em: 03/09/2021

\footnotetext{
${ }^{1}$ Docteur en épistémologie et histoire des sciences par 1'Université Paris Diderot. Chargé de recherche au Centre national de la recherche scientifique (CNRS) Membre du laboratoire SPHERE (UMR 7219, CNRS \& Université Paris Diderot). Adresse de correspondance: 25 rue du plateau, 91360 Épinay sur Orge, France. E-mail: eric.vandendriessche@u-paris.fr

2 Doutora em Educação pela Universidade de São Paulo. Professora do Programa de Pós-Graduação em Educação da Universidade Federal Fluminense. Endereço para correspondência: Rua Bolívar 163/301, Rio de Janeiro, RJ, Brasil, CEP: 22061020. E-mail: mc_fantinato@id.uff.br
} 
circunstâncias de sua primeira colaboração. A discussão então se volta para o desenvolvimento da etnomatemática no Brasil e na Europa, bem como para as relações desse campo de pesquisa com a antropologia, a epistemologia e a educação. Por fim, os dois etnomatemáticos se questionam sobre os desafios atuais para a etnomatemática como área de pesquisa internacional.

Palavras-chave: Etnomatemática; Diálogo; Educação; Brasil; Europa.

\title{
Ethnomathematics in Brazil and Europe: a dialogue
}

\begin{abstract}
On occasion of this special issue of the Journal REMat devoted to ethnomathematics in its links with the "decolonization and resistance movements", Brazilian ethnomathematician and educator Maria Cecilia Fantinato and French ethnomathematician Éric Vandendriessche decided to contribute to this volume with a dialogue on the topic of ethnomathematics. The discussion transcribed in this article thus addresses some issues related to the development of this young interdisciplinary field in different contexts. The two researchers first explain the circumstance of their first collaboration. The discussion then turns to the development of ethnomathematics in Brazil and Europe, as well as the relationship between this field of research and the anthropology, epistemology and education. Finally, they raise some of the current challenges for ethnomathematics as an international research area.
\end{abstract}

Keywords: Ethnomathematics; Dialogue; Education; Brazil; Europe.

\section{En guise d'introduction}

Nous avons souhaité contribuer à ce volume - consacré à l'ethnomathématique - sous la forme d'un dialogue. Le 28 août 2020, nous avons enregistré une longue discussion organisée sur Skype autour de quelques thèmes choisis, tout en laissant notre conversation s'aventurer vers des sujets imprévus. Le texte qui suit est le résultat de la retranscription de ce dialogue.

\section{Chemins croisés vers l'ethnomathématique}

MC: Nous avons des parcours différents qui nous ont néanmoins mené tous les deux à l'ethnomathématique. Je propose pour commencer notre discussion que nous rappelions nos parcours. Alors, Éric, quelle a été la route qui t'a mené à l'ethnomathématique ?

E: C'est une longue histoire. Ma formation initiale a été les mathématiques. J'ai ensuite obtenu 1'“Agrégation" et enseigné les mathématiques pendant une vingtaine d'années dans le secondaire. Tout au long de mes années d'enseignement, je n'ai jamais cessé de m'interroger sur la nature et l'histoire des savoirs que j'enseignais, et sur la façon dont s'est constituée cette discipline. Réflexion que je partageais avec mes élèves. En particulier, j'ai collaboré un temps avec l'Institut de recherche sur l'enseignement des mathématiques (IREM) pour réfléchir à l'usage de textes de 
mathématiciens pour enseigner les concepts mathématiques en les replaçant dans leur contexte historique.

MC: Etais-tu déjà allé au Vanuatu?

E: Non, pas encore. Mais, en effet, il y a 30 ans, j'ai interrompu ma carrière en France pour faire ce que l'on appelait à l'époque le service national de la coopération. J'ai ainsi été au Vanuatu ${ }^{3}$, dans le Pacifique sud, pour la première fois en 1989 pour enseigner pendant deux ans les mathématiques dans un collège francophone de l'île d'Espiritu Santo. Cette expérience a été d'une grande importance dans mon parcours, et tout particulièrement la rencontre avec des sociétés autochtones dans lesquelles j'avais été introduit par certains de mes élèves. J'ai été séduit par ces gens, par leur mode de vie, leur économie autosuffisante, même si je ne pensais pas du tout à l'époque que je m’intéresserai un jour aux savoirs mathématiques qu'ils développent ou ont développé.

Des années plus tard, à un carrefour de ma carrière, dans les années 2000, j'ai souhaité faire autre chose que d'enseigner les mathématiques. Un congé de formation d'une année m'a offert l'opportunité de me consacrer à plein temps à un Master d'histoire et philosophie des sciences, avec le projet de développer des recherches à la croisée des mathématiques et de l'anthropologie. Je me suis alors pris au jeu, trouvant très intéressant l'étude de la façon dont se constituent les savoirs scientifiques dans différents contextes historiques, sociaux, et culturels. J'ai ensuite rencontré la personne qui allait devenir ma directrice de thèse, la Pr. Karine Chemla, historienne des mathématiques, spécialiste de la Chine ancienne, à qui j’ai posé la question de savoir si certaines sociétés ou communautés pratiquaient (ou auraient pratiqué) les mathématiques uniquement de manière orale, sans le support de l'écriture. Elle m'avait alors conseillé de lire le premier ouvrage de Marcia Ascher (1991), dont elle venait de publier une traduction en français avec l'anthropologue Serge Pahaut (1998). La lecture de ce livre, et celle du chapitre traitant des dessins sur le sable de Vanuatu en particulier, mais aussi la conclusion écrite par Karine Chemla et Serge Pahaut (soulignant l'importance d'étudier les mathématiques impliqués dans nombre de pratiques au-delà des champs savants et institutionnels), m’a passionné et convaincu que c'était là le domaine dans lequel je souhaitais inscrire mon travail. C'est donc dans la continuité de ce travail novateur de Marcia Ascher que j'ai fait mes premiers pas dans le champ de l'ethnomathématique. J'ai ensuite fait une thèse de doctorat consacrée à pratique des jeux de ficelle - dirigée par Karine Chemla et l'anthropologue des techniques Sophie Desrosiers, qui m’ont accompagné dans mes premières années de chercheurs. J'ai ensuite eu la chance de pouvoir continuer à mener mes recherches en France au Centre National de

${ }^{3}$ La République de Vanuatu est un archipel du Pacifique Sud, anciennement connu sous le nom de Nouvelles-Hébrides, géré par le condominium colonial franco-britannique de 1906 jusqu'à son indépendance en 1980. 
la Recherche Scientifique (CNRS), dans un domaine qui était jusqu'ici peu développé dans ce pays, nous en parlerons plus tard. Et toi donc, quel a été ton chemin vers l'ethnomathématique?

MC: En t'écoutant, je pensais à mon propre chemin. Bien qu'il soit différent, il y a des similitudes. Je ne suis pas mathématicienne, mais en tant que psychopédagogue, je me suis toujours intéressée à l'apprentissage des mathématiques. Dans mon parcours professionnel, qui a débuté par un poste d'enseignante à l'école primaire, j'ai été influencée par les théories de Piaget et de ses disciples, comme Constance Kamii. Néanmoins, ces travaux ne répondaient pas à mes interrogations sur les relations entre les apprentissages et le contexte socioculturel. Le travail de Carraher, Carraher et Schliemann (1989) m'a ensuite beaucoup inspiré. Il examine les liens entre les connaissances mathématiques d'enfants travaillant sur les marchés - connaissances acquises et pratiquées dans des activités de la vie quotidienne en dehors de l'école -, et celles enseignées tout au long du processus scolaire. C'est avec ce profil que je suis devenue professeur à l'Université Fédérale Fluminense (UFF) en éducation mathématique pour les futurs enseignants et les pédagogues. En 1997, j’ai été invitée par une amie - Sonia de Vargas - à enseigner dans un cours (à l'UFF) de spécialisation sur l'éducation des adultes. Je n'avais alors aucune expérience dans ce domaine.

J'ai donc engagé une recherche exploratoire: cela m'a beaucoup intéressé d'observer la façon dont ces gens procédaient pour calculer, et d'écouter leurs commentaires sur ce qu'ils étaient en train d'apprendre avec leur professeur. Je cherchais à comprendre le point de vue de ces adultes peu scolarisés; il y avait une logique dans leur pratique, qui parfois n'était pas celle des savoirs scolaires, choisissant d'autres chemins, impliquant le recours au calcul mental ou aux brouillons notamment.

Cette expérience a été mes premiers pas vers mon projet de doctorat. C'est à cette période que j'ai rencontré l'ethnomathématique dont j'ai entendu parlé par des collègues en éducation mathématique. Je me souviens avoir assisté, en 1998, à une table ronde consacrée à l'ethnomathématique ${ }^{4}$, à laquelle participaient plusieurs chercheurs brésiliens de ce domaine, parmi eux figuraient Ubiratan D’Ambrosio, Gelsa Knijnik, Eduardo Sebastiani Ferreira, et ma future directrice de thèse, Maria do Carmo Domite.

Je suis alors entrée à l'université de São Paulo en 1999. C'était un moment pionnier, parce que Maria do Carmo Domite, encouragée par D’Ambrosio, venait de constituer le premier groupe de recherche en ethnomathématique au Brésil (GEPEm). Au cours de l'année 2000, ce groupe a organisé le premier congrès brésilien d'ethnomathématique $(\mathrm{CBEm} 1)$. Tout ça a fait partie de ma formation: les discussions aux réunions du GEPEm, le cours d'histoire des sciences donné par D'Ambrosio, les échanges avec ma directrice de thèse, la période de stage doctoral à l'Université Rutgers avec Arthur

${ }^{4}$ 6e Encontro Nacional de Educação Matemática (VI ENEM), en 1998. 
Powell, et la collaboration de Marcio D’Olne Campos, physicien devenu anthropologue, qui réfléchit de façon critique aux ethnosciences. J'ai été beaucoup influencé par les idées de Marcio sur l'"ethnographie des connaissances techniques et pratiques" (CAMPOS, 2001), qui m'ont permis d'élargir mon regard de chercheuse, de passer - tout au long du travail de terrain - d'une vision restreinte des mathématiques et une vision plus élargie. Mon travail de thèse a impliqué une recherche ethnographique dans la communauté de São Carlos (FANTINATO, 2004), qui visait à mieux comprendre les processus et les connaissances (mathématiques) des jeunes et des adultes, élaborées dans des contextes de la vie quotidienne, ainsi que ses possibilités d'articulation avec les connaissances scolaires.

E: C'est effectivement un point dont nous pourrons reparler plus loin, la relation entre l'ethnomathématique et l'anthropologie/ethnographie, qui a compté dans notre rencontre, lorsque tu es venue vers moi il y a quelques années sur une suggestion d'Ubiratan D’Ambrosio.

MC: Oui, c'est quelque chose qui nous rapproche.

E: Nous avons tous les deux souhaités aller au-delà des premières études ethnomathématiques des années 1980-90, voire 2000, en menant nos propres recherches de terrain, pour rencontrer des acteurs/praticiens/experts, plutôt que de mener nos travaux uniquement sur la base de publications secondaires (et non d'études directes) comme le faisait Marcia Ascher entre autres.

MC: Et pas seulement cela. Il y a beaucoup de recherches menées au Brésil, pour lesquelles les chercheurs font du terrain, mais avec une approche un peu différente. Ils vont sur le terrain avec leur conception des mathématiques, et ne sont pas assez ouverts pour regarder comment les gens pensent les relations entre leurs mathématiques et d'autres aspects de la culture, la religion, les activités quotidiennes, etc.

J'ai fini mon doctorat et je suis retournée à mes activités à l'université. En 2004, j'ai collaboré avec la municipalité de Rio, qui avait lancé un programme d'éducation pour les jeunes adultes (PEJA). Alors, une année après mon doctorat, j'ai commencé à travailler comme conseillère pour le PEJA, dans le cadre de la formation continue des professeurs de mathématiques qui s'occupaient de ces étudiants particuliers. Cela a été une vraie rencontre. Plusieurs de ces professeurs, ayant l'expérience des classes pour adultes, avaient pris conscience que ces étudiants savaient beaucoup de choses, mais d'une façon différente. Il fallait apprendre avec eux, en établissant un dialogue pour réflechir à l'éducation adaptée à ce public très particulier. L'ethnomathématique leur semblait être une approche théorique intéressante pour comprendre les processus et les connaissances (mathématiques) de ses étudiants adultes. Certains de ces enseignants, comme mon premier étudiant de master, André Luiz Gils, ont participé à la création de mon groupe de recherche en ethnomathématique (GETUFF) en 2004. 
E: Je propose que nous évoquions notre première collaboration. Cela nous permettra de faire une transition vers la question du développement de l'ethnomathématique au Brésil que nous souhaitions aborder.

\section{Ethnomathématique au Brésil}

E: J'étais en contact avec Ubiratan D'Ambrosio, et en tant que jeune éthnomathématicien j'étais très intéressé par le développement des recherches ethnomathématiques au Brésil, développement impressionnant en comparaison avec d'autres pays. Si ce type de recherche a également été développé aux USA, et sur les continents australiens et africains notamment, il s'est de fait passé quelque chose d'assez singulier au Brésil. Par ailleurs, il me semblait regrettable de ne pas avoir accès à nombre de ces travaux ne parlant ni ne lisant correctement le portugais, langue dans laquelle sont écrites la plupart des contributions brésiliennes en ethnomathématique. Dans la perspective de combler cette lacune, j'ai préparé un projet européen Marie-Curie avec l'idée de séjourner deux ans au Brésil pour enrichir mes connaissances en ethnomathématique des avancées brésiliennes dans ce domaine, apprendre le portugais, etc. J'en ai informé D'Ambrosio - qui connait bien mon travail, et à qui je suis très reconnaissant de m'avoir soutenu tout au long de ces dernières années -, en lui demandant conseil pour identifier un.e "adviser" brésilien.ne. Quelque temps plus tard, tu m'écrivais pour me dire ton intérêt pour mes recherches et me proposer de collaborer à la rédaction de ce projet de mobilité vers le Brésil.

MC: Oui, c'est cela, tu cherchais des collaborateurs.

E: Oui, dans le cadre de ce projet Marie-Curie, deux "advisers" devaient encadrer mon travail, l'un.e au Brésil et l'autre en Europe. C'est la philosophe belge Karen François, que tu connais bien, qui devait jouer ce rôle en Europe. Mon projet n’a finalement pas été sélectionné, mais sa préparation aura permis d'initier notre collaboration, tout en commençant à s'interroger sur les raisons pour lesquelles l'ethnomathématique s'était développé de cette façon au Brésil, et sur les différences avec les lignes de recherche développées (peu, il est vrai) en Europe. J'étais tout particulièrement intéressé par les relations que l'ethnomathématique entretient avec l'éducation mathématique, et les questions politiques, didactiques, pédagogiques soulevées par ces recherches interdisciplinaires. Plus tard, j'ai appris dans tes travaux (FANTINATO, 2013) que l'éducation est le domaine le plus travaillé par l'ethnomathématique au Brésil (voire au-delà). Je percevais que dans ce pays était menée une réflexion approfondie sur les questions d'éducation mathématique, résultant probablement (pour partie) des revendications des sociétés autochtones brésiliennes pour la prise en compte de leur culture dans les programmes scolaires; mais pas seulement, la preuve en est ton travail sur l'éducation des jeunes adultes. 
MC: D'Ambrosio a eu une belle intuition en me proposant de venir vers toi. Peut-être est-ce aussi parce que j'avais déjà un réseau de contacts internationaux à cette époque là - constitué avec l'aide de Maria do Carmo Domite. J'avais également organisé un congrès important, le $3 \mathrm{e}$ "Congrès Brésilien d'ethnomathématiques" (CBEm3), et édité le livre regroupant les principales interventions de cet évènement (FANTINATO, 2009). Cela a contribué à me positionner sur le plan national dans ce domaine.

\section{Du Brésil à l'Europe}

E: Oui, D’Ambrosio a eu une bonne intuition. Une question au cœur de ton nouveau projet, sur lequel nous avons souvent échangé, concerne plus précisément la comparaison du développement de l'ethnomathématique entre le Brésil et l'Europe, et les influences mutuelles. A ma connaissance, peu de chercheurs européens mènent des recherches dans ce champs - et, comme au Brésil, il s'agit le plus souvent de travaux en lien avec l'éducation mathématique. En France néanmoins, c'est plutôt dans le champ de l'anthropologie que ce champ de recherche se développe - du fait de mon recrutement au CNRS en section 38 "Anthropologie et étude des sociétés contemporaines" notamment. Si quelques didacticiens français s’y intéressent (BULF 2010, CASTELLA; ELGUERO 2013), l'ethnomathématique tend à devenir en France une sous discipline de l'anthropologie. Sur le plan institutionnel, j'ai été recruté pour mener des travaux ethnomathématiques dans ses liens avec l'anthropologie et non avec l'éducation mathématique, même si ces questions m'intéressent compte tenu de mon parcours. C'est d'ailleurs sur ces questions que portent notre collaboration avec Karen François et José Mafra (VANDENDRIESSCHE et al. 2017, FRANÇOIS et al. 2018), nous en reparlerons.

Quoiqu'il en soit le développement de l'ethnomathématique en Europe est beaucoup plus lent qu'il ne l'a été au Brésil, et a commencé beaucoup plus tardivement.

MC: nos premiers échanges datent de 2013, je me souviens. J'avais déjà publié ce travail sur les différents champs disciplinaires représentés dans les congrès d'ethnomathématique au Brésil (FANTINATO, 2013). C'était le premier travail où j'ai commencé à concevoir 1'ethnomathématique comme un véritable domaine de recherche. Je me suis alors aperçue - même si cela ne m'a pas vraiment étonné - que les recherches ethnomathématiques touchant à l'éducation étaient de plus en plus nombreuses dans mon pays. Au commencement, il y avait surtout des recherches de type “ethnographique”, et, au fur et à mesure, l'intérêt des ethnomathématiciens s'est porté sur l'éducation. Je pense que l'histoire de l'ethnomathématique au Brésil est différente de celle des autres pays. Elle est très liée à D'Ambrosio, comme l'a montré le travail de mon ex-doctorant Fabio Lennon Marchon (2019). Il a étudié de façon approfondie le récit historique de l'ethnomathématique au Brésil dans ses 
liens avec les premiers livres de D'Ambrosio $(1990,2001)$ et leur influence sur la production des chercheurs brésiliens.

Tu précisais qu'en France l'ethnomathématique devenait une sous-discipline de l'anthropologie. Au Brésil ce n'est pas du tout le cas. L'ethnomathématique a été ici associée à la constitution de l'éducation mathématique en tant que domaine scientifique (PASSOS, 2017).

D’Ambrosio a eu un rôle important dans la formation des associations liées à l'ethnomathématique. L'une des premières a été la "Société Brésilienne d'Éducation Mathématique" (SBEM), dont l'ethnomathématique était l'une des lignes de recherche. Sous l'influence de D'Ambrosio, le premier numéro de la revue éditée par cette société fut dédiée à l'ethnomathématique ${ }^{5}$, ce qui explique pour partie la relation de l'ethnomathématique avec l'éducation.

Au-delà de D'Ambrosio, on trouve dans les années 1990, des mentions à l'ethnomathématique dans des documents officiels sur l'éducation, comme les "Parâmetros Curriculares Nacionais". Il y a également eu la loi № 11.645 de 2008, visant à mettre en œuvre une éducation scolaire en relation avec la culture et l'histoire des peuples autochtones et d'origine africaine, et prendre en compte notre diversité culturelle.

Cette loi qui a été créée en réponse à des mouvements sociaux, et en guise de réparation historique, a eu un réel impact sur l'école. Beaucoup d'enseignants ont rencontré l'ethnomathématique à ce moment là, pour enrichir leur enseignement des influences africaines dans la culture brésilienne.

Dans des congrès brésiliens on trouve de nombreux travaux sur l'utilisation de jeux africains - comme le mancala - dans les classes de mathématiques. Cela est une conséquence de cette loi. Il y a un intérêt croissant pour les recherches portant sur les "africanités", et dans le champ de l'ethnomathématique notamment (SILVA; COSTÄ, 2018). Un livre récemment publié sur la recherche brésilienne en ethnomathématique (ROSA; OLIVEIRA, 2020) comporte une partie entière sur le thème des "africanités", et un autre sur la "diversité indigène" ("indigenous diversities"). Au Brésil, l'ethnomathématique est donc profondément liée à l'éducation et de plusieurs façons. Un autre exemple est celui des cours "Licenciaturas Interculturais indígenas" à destination des enseignants autochtones dans lesquels ont retrouve les ethnomathématiques liées à la formation des éducateurs (SURUÍ; LEITE, 2018; OLIVEIRA; MENDES, 2018).

E: C'est ce qui nous a incité à collaborer ces dernières années avec Karen François et José Mafra; cette relation entre l'ethnomathématique et l'éducation; et, plus précisément, l'usage de

\footnotetext{
${ }^{5}$ Educação Matemática em Revista N. 1, 1993.

http://sbem.iuri0094.hospedagemdesites.ws/revista/index.php/emr/issue/view/140
} 
savoirs informels dans le contexte scolaire - qui ne va pas de soi et pose différentes questions que nous avons travaillées dans nos articles collaboratifs. Il s'agit de questions de nature épistémologique qu'implique la rencontre de différents types de savoirs. Cela nous mène au point suivant que nous souhaitions aborder dans cette conversation.

\section{Approches critiques de l'ethnomathématique}

E: Il existe des critiques sévères et pertinentes sur l'usage de savoirs informels dans le cadre formel (à l'école par exemple), et pour des enseignements culturellement situés à destination des sociétés autochtones notamment. Je pense en particulier aux écrits du philosophe Alexandre Pais (2011) qui a soulevé le risque de décontextualisation des savoirs traditionnels. Par exemple, l'ethnomathématicien Paulus Gerdes a cherché à mettre en évidence le théorème de Pythagore dans des vanneries du Mozambique (1992), tentative qui nous éloigne significativement du point de vue des acteurs. Le rapport à la culture en devient de fait très ténu.

Dans nos travaux collaboratifs $(2017,2018)$, nous avons cherché à répondre à cette critique en proposant que des activités à caractère algorithmique et/ou géométrique (comme les jeux de ficelle, les dessins sur le sable, la fabrication/décoration de gourdes) soient pratiquées pour elles-mêmes, sans chercher à y déceler des théorèmes provenant d'autres cultures mathématiques, et en amenant dans la classe toute la complexité culturelle et cognitive de ces pratiques.

Si ces questions éducatives étaient assez loin de mes premières préoccupations de chercheur, j'ai été fortement incité par les institutions locales de Vanuatu (le Centre Culturel de Vanuatu en particulier) à participer aux discussions sur les questions relatives à l'usage de savoirs traditionnels pour enseigner les mathématiques d'une façon différente (cf. VANDENDRIESSCHE et al. 2017). Il y a une volonté politique forte au Vanuatu - et dans nombre de sociétés océaniennes - pour que des enseignements culturellement signifiant soient mis en place dans le système scolaire.

MC: Je pense que c'est un peu notre rôle de chercheurs en ethnomathématique, sans nier nos différences, mais en cherchant à s'aider mutuellement. Ces populations sont en demande de cela. Mafra a vécu la même chose avec les artisanes de Pará qui lui proposèrent que les savoirs impliqués dans la fabrication de gourdes soit inclus dans les curriculum des écoles locales. Notre petit groupe de recherche (Karen, Mafra, toi, et moi-même) cherche à mieux comprendre la complexité de cette relation: par exemple en interrogeant la place du chercheur sur le terrain devenant l'autre ("concept of the Other").

En 2015-2016, j'ai supervisé le travail de post doctorat de José Ricardo Mafra (2016). Il est mathématicien, mais il avait déjà fait du terrain, un peu à la façon de Paulus Gerdes. Mafra regardait les dessins, les symétries, dans les marques dessinées sur les gourdes. Il me semble l'avoir aidé à 
élargir son point de vue. Dans cette perspective, je l'ai accompagné sur le terrain : nous avons regardé au-delà des motifs géométriques, en étudiant les modes de production, les relations avec l'économie locale, les interactions entre ces femmes/praticiennes; ainsi que les processus d'apprentissage et de transmission de ces savoirs (DE VARGAS, 2009).

Il me semble que les recherches ethnomathématiques sont encore parfois aujourd'hui trop restrictives, conduisant trop rapidement vers des modélisations. C'est plus ou moins la critique que fait Pais, qu'en penses-tu?

E: Oui, certainement. Néanmoins, ce que tu dis sur la modélisation me fait penser à la conversation entre Ascher et D'Ambrosio publiée dans la revue "For the Learning of Mathematics" (1994). De fait, la modélisation des savoirs (ethno)mathématiques est la méthodologie introduite par Marcia Ascher dans les années 1980. Loin des questions éducatives, elle commença par étudier les khipu Inca (1981). Ascher explique à D'Ambrosio qu'elle préférait ne rien connaître de la culture Inca, en étudiant d'abord les khipu de façon formelle, en cherchant à comprendre - avec les outils dont elle disposait - les logiques de ces artefacts. Néanmoins, elle explique plus loin que la collaboration avec son mari Robert Ascher, anthropologue, a permis, par la suite, de mettre en évidence que ces logiques faisaient écho à des règles et des structures sociales dans la société concernée.

J'ai eu cette discussion avec Marc Chemillier il y a quelques années (VANDENDRIESSCHE, 2015), dans le cadre d'un numéro de la revue de sciences humaines ethnographiques.org (LAMBELET et al., 2015). Ce qui intéresse les anthropologues dans l'ethnomathématique c'est justement les interrelations entre des pratiques (ethno-)mathématiques et d'autres phénomènes sociaux au sein de la même société. Étudier les aspects formels de ces activités sans regarder comment elles s'insèrent dans la société apparaît généralement comme très insatisfaisant aux yeux des anthropologues. Comme Ascher, Chemillier défend la position contraire, en affirmant que les aspects formels d'une activité méritent d'être étudiés pour eux-mêmes. C'est ce que fait Chemillier dans ses travaux sur la divination malgache (sikidy), en analysant la façon dont les devins manipulent des matrices de graines (2007).

\section{Ethnomathématique sur le terrain}

E: Si, dans une certaine mesure, je partage l'opinion de Chemillier, il me semble néanmoins important de saisir ce que ces analyses formelles de pratiques à caractère mathématique permettent de dire de la société étudiée. C'est ce que j'ai cherché à faire en collaborant avec l'ethnologue Céline Petit, avec qui je travaille depuis une dizaine d'années. Dans le cadre de deux projets collectifs 
financés ${ }^{6}$ portant sur la pratique des jeux de ficelle (Océanie, Amérique du Nord et du Sud), nous avons systématiquement croisé nos propres perspectives disciplinaires. La rencontre de différents champs disciplinaires est l'une des caractéristiques de l'ethnomathématique qui est par nature un domaine interdisciplinaire. Conduire des recherches interdisciplinaires n'est pas si simple. Cela a pris du temps pour construire une méthodologie commune, élaborer de nouveaux projets de recherche, et produire des articles scientifiques. Pour étudier ces pratiques avec une double approche ethnomathématique et anthropologique il nous a semblé essentiel d'avoir systématiquement recours à l'ethnographie, en menant un travail de terrain, et ainsi rencontrer des praticiens en "chair et en os" dans la perspective de mieux saisir leur point de vue sur ces activités, et engager un dialogue avec ces experts.

MC: Mais toi, tu t'intéresses aux aspects formels des jeux de ficelle, mais aussi à ce que cette activité représente pour ces gens-là; aux histoires qui accompagnent la réalisation de ces figures par exemple. Comment fais-tu dialoguer ces deux aspects?

E: Quand je suis allé sur le terrain pour la première fois dans les îles Trobriand, en PapouasieNouvelle-Guinée, pour y étudier la pratique des jeux de ficelle (cf. VANDENDRIESSCHE 2012, 2015b) - j’ai engagé un travail de collecte des procédures que je découvrais. Je me suis néanmoins rapidement rendu compte que cette pratique s'ancre dans une réalité sociale et culturelle complexe qui lui donne sens. J'ai donc collecté d'autres données ethnographiques dans la perspective d'étudier la façon dont les procédures ou les transformations impliquées dans ces jeux de ficelle font écho à d'autres phénomènes culturels et sociaux dans la société trobriandaise. L'enjeu était ici d'analyser cette activité dans toutes ses dimensions cognitives et culturelles.

Les méthodes de modélisation que nous utilisons ensuite pour modéliser les jeux de ficelle permettent de mettre au jour des systèmes de transformation, des interconnexions/relations entre les procédures de jeu de ficelle d'un même corpus, ou d'un corpus à l'autre. Et ainsi de faire des hypothèses sur la façon dont des acteurs ont créé ces jeux de ficelle dans le passé, en lien avec des contextes culturels spécifiques.

MC: Mais les acteurs le perçoivent de façon différente, n'est-ce pas ?

E: Oui, tu as raison, les acteurs perçoivent ou expriment ces phénomènes le plus souvent de façon différentes. Néanmoins, si la modélisation de ces pratiques mathématiques relève d'un point de

\footnotetext{
6 Projet "Les Jeux de ficelle: aspects culturels et cognitifs d'une pratique à caractère mathématique" (2012-2016) http://www.sphere.univ-paris-diderot.fr/spip.php?rubrique84

Projet ANR "Encoding and Transmitting Knowledge with a String : a comparative study of the cultural uses of mathematical practices in string-figure making (Oceania, North \& South America)" (ETKnoS) (2016-2020) http://www.sphere.univ-paris-diderot.fr/spip.php?rubrique153
} 
vue d'observateur, elle permet de mettre en évidence certaines régularités ou propriétés d'un corpus donné. De plus, par cet exercice, le modélisateur acquiert la maîtrise des procédures en jeu dans ces pratiques, ce qui s'avère ensuite très utile sur le terrain pour être attentif à certains détails ou variantes dans la façon dont opèrent les acteurs.

MC: Peut-être serait-il intéressant d'établir un dialogue avec un praticien qui pourrait parler de son point de vue et toi du tien à propos de ces pratiques. As-tu déjà essayé de faire cela?

E: Oui, ça peut fonctionner, on peut chercher à échanger nos points de vue...

MC: Dans l'interview que tu as réalisée de Marc Chemillier (cf. VANDENDRIESSCHE, 2015a) vous évoquez ces échanges avec les acteurs locaux.

E: Oui, il raconte que - "si l'on fait le travail de maîtriser une technique" - la relation entre le praticien et le chercheur devient une relation de "confrère à confrère". Il ne s'agit plus alors d'une simple observation-participante, le chercheur est traité par l'expert "comme un collègue", puisque le chercheur partage avec le praticien des connaissances que les autres n'ont pas. Cela "facilite beaucoup l'enquête, dont de nombreux aspects sont liés à cette interrelation assez particulière qui s'établit'.

\section{Ethnomathématique et anthropologie}

MC: Une chose dont nous avons déjà parlé, et que nous devrions discuter plus loin, est la relation de l'ethnomathématique avec l'anthropologie. Il y a quelques temps, tu me demandais si l'anthropologie - qui est un champ de recherche très développé au Brésil - avait eu une influence sur le développement des ethnomathématiques que l'on a connu dans ce pays. Selon moi, cette influence est limitée. Il n'y pas eu vraiment de rencontre entre ces deux champs disciplinaires.

Lorsque nous avons participé ensemble au congrès de l'IUAES à Florianópolis en 2018, dans le Panel "Indigenous Mathematical Knowledge"7, que tu organisais avec Rik Pinxten et Céline Petit, il y avait des anthropologues et des ethnomathématiciens. Lorsque j'ai parlé des domaines de recherche en ethnomathématique, je me suis aperçue que les anthropologues brésiliens présents ne connaissaient rien à ce domaine. Probablement, que la raison vient du fait qu'au Brésil ce dernier est plutôt liée à l'éducation mathématique. La relation anthropologie-ethnomathématique est peu développée. Si certains anthropologues étudient des sujets proches, comme les savoirs autochtones que l'on peut associer aux mathématiques, ils ne semblent pas connaitre les recherches ethnomathématiques. Il serait bien que l'on puisse se rencontrer et développer des échanges entre ces deux champs disciplinaires.

\footnotetext{
7 http://www.inscricoes.iuaes2018.org/trabalho/view?ID TRABALHO=508
} 
E: Cette question de la relation entre le développement de l'ethnomathématique et celui de l'anthropologie au cours du $20^{\mathrm{e}} \mathrm{s}$, nous l'avons abordé dans un article historiographique sur l'ethnomathématique (2017) que j'ai coécrit avec Céline Petit. Cet article montre que les prémices d'une anthropologie des mathématiques sont perceptibles dans plusieurs études ethnologiques réalisées à partir du $19^{\mathrm{e}} \mathrm{s}$. siècle. Néanmoins, c'est véritablement l'entrée dans la seconde moitié du 20e siècle qui semble marquer un tournant épistémologique quant à la façon dont les anthropologues aborderont le thème des formes logiques manifestées dans les sociétés non-occidentales. Et d'ailleurs, c'est en prenant plus significativement appui sur des travaux de l'anthropologie que certain(e)s mathématicien(ne)s - comme Marcia Ascher - mèneront des travaux aujourd'hui considérés comme fondateurs de l'ethnomathématique.

Etant donné l'importance de l'anthropologie brésilienne sur le plan international, se posait la question de savoir si le développement de cette discipline aurait pu avoir une incidence sur celui de l'ethnomathématique et de son institutionnalisation dans ce pays. Et, finalement, comme tu le signalais, il n'y aurait pas de relation directe entre ces deux domaines de recherche qui dialoguent assez peu au Brésil.

MC: Oui, j'en avais l'intuition, et maintenant j'en suis certaine. Cela fait maintenant une vingtaine d'années que je mène des recherches dans ce domaine. J'ai participé aux différents congrès nationaux et internationaux sur l'ethnomathématique. De plus, avec mon collègue Kécio Leite, nous avons étudié la production ethnomathématique brésilienne consacrée aux sociétés autochtones (FANTINATO; LEITE, 2020). J'ai donc maintenant une idée générale du développement de l'ethnomathématique au Brésil.

Une autre chose que je voulais mentionner est le rôle important des groupes de recherche dans les universités brésiliennes. Lorsque j'ai fait mon doctorat à l'université de São Paulo, il y avait peu de groupes de ce genre, mais des chercheurs formés à l'ethnomathématique, originaires d'autres régions du Brésil, ont initié des groupes de recherche dans leur région, par exemple dans le nord en Amazonie. Ces groupes ont été créés un peu partout au Brésil, et ce phénomène reste très marqué dans les régions sud/sud-est. D'un groupe à l'autre, il y a néanmoins de petites différences concernant les sujets de recherche abordés. Par exemple, au Nord et au Centre-Ouest les populations autochtones sont plus nombreuses; ce qui semble entraîner un plus grand intérêt pour les questions d'éducation indigène ou celle des quilombola. De la même façon, dans l'état de Pará, il y a des populations riveraines, et dans cette région sont menés davantage de travaux sur les savoirs et l'éducation de ces groupes (FORMIGOSA, LUCENA \& SILVA, 2017).

Au Sud-Sud Est, on trouve d'autres tendances. Notamment des groupes de recherche qui ont des approches philosophiques - inspirées des œuvres de Wittgenstein, Foucault, Derrida. Les travaux 
du groupe de Gelsa Knijnik à UNISINOS, de Roger Miarka à l'UNESP, Jackeline Mendes, Denise Vilela et Alexandrina Monteiro à l'UNICAMP en sont des exemples. Bien sûr, on trouve des travaux en lien avec la philosophie également dans d'autres régions du Brésil, mais de façon moins prononcée.

Dans mon propre groupe à l'UFF, nous avons commencé par mener des travaux sur l'éducation des jeunes adultes, et poursuivi des recherches sur ce thème jusqu'à aujourd'hui. Un exemple est le travail de maîtrise de Claudia Meira (2015) qui a étudié les savoirs mathématiques d'adultes privés de liberté, et les relations entre ces connaissances et celles acquises à l'école. Néanmoins, petit à petit, le groupe s'est ouvert à d'autres sujets, en lien avec les recherches de mes étudiants: par exemple Fabio Lennon qui a étudié - dans le cadre d'un master - les approches philosophiques en ethnomathématique (MARCHON; FANTINATO, 2015), et mené ensuite des études doctorales visant à analyser les contributions de D'Ambrosio à la lumière des travaux de Paul Ricœur.

\section{De l'épistémologie à l’éducation}

E: Cela nous ramène aux questions épistémologiques (voire philosophiques) liées à la reconnaissance de différentes pratiques comme relevant des mathématiques. Il me semble que nous devons maintenant dépasser cette question. De mon point de vue, la bonne question est plutôt de savoir ce que l'on gagne sur le plan épistémologique à élargir notre point de vue sur les mathématiques, en y incluant l'ensemble des activités à caractère "arithmétique", "géométrique", ou encore "algorithmique" (tressage, divination, navigation, jeux, musique, etc.), pratiquées dans le passé et/ou de nos jours dans diverses sociétés.

Je pense que nous aurions beaucoup à gagner en suivant le mouvement engagé récemment par certains historiens des sciences qui ont travaillé sur le concept de preuves mathématiques en particulier (Chemla, 2012). Plutôt que de restreindre le cadre de l'étude aux raisonnements hypothético-déductifs, ils ont cherché au contraire à élargir ce concept, en y incluant toutes les pratiques (les corrections d'algorithmes dans les mondes anciens notamment) pour lesquels on décèle de la part des acteurs une intention de justification.

Dans le même ordre d'idée, il me semble que nous devrions chercher à dépasser l'opposition entre mathématiques "occidentales" et "non-occidentales" souvent avancée dans les recherches ethnomathématiques (Radford, 2020). De fait, les travaux d'histoire des mathématiques menés depuis quelques décennies (par des historiens du laboratoire SPHERE auquel j'appartiens notamment) ont mis au jour la circulation des savoirs mathématiques entre ces différents mondes, ce concept de "mathématiques occidentales" devenant de fait assez obscure selon moi. 
Si ces questions de nature épistémologique ne forment pas le cœur de ton travail, elles inspirent tes recherches, n'est-ce pas?

MC: Oui, toutes ces recherches ethnomathématiques inspirent mon travail et mes enseignements, celui consacré à la pédagogie notamment. Les étudiants ont parfois vécu de mauvaises expériences avec les mathématiques dans leur cursus scolaire, et, pour cette raison, peuvent avoir quelques inquiétudes pour les enseigner. Alors je leur montre qu'il peut y avoir plusieurs façons de résoudre des problèmes mathématiques; par exemple, différentes méthodes pour effectuer des divisions. Cela rassure ces étudiants. De fait l'école, comme institution, a tendance à proposer un curriculum homogène. Il faut donner davantage de place à la diversité de pensée, et tout particulièrement dans le cours de mathématiques.

La réflexion sur les curriculums menée par l'ethnomathématique permet de mieux saisir la complexité du dialogue entre différents types de savoirs dans le contexte scolaire (MONTEIRO; MENDES, 2014). L'une de mes étudiantes, Gisele Américo Soares, termine un travail doctoral sur les apports de l'ethnomathématique dans la formation initiale des futurs professeurs de mathématiques. L'introduction à ce domaine de recherche permettrait d'élargir le point de vue de ces étudiants sur la nature des mathématiques dans ses relations avec d'autres domaines de connaissances.

J'ai une question à te poser en lien avec mon projet actuel. Tu ne t'intéresses pas vraiment à l'éducation, ce n'est pas ton sujet. Mais, tu sais qu'il y a des contributions sur ce thème en Europe, en relation avec l'enseignement à destination des classes multiculturelles, les réfugiés, etc. Comment vois-tu cette question qui est de plus en plus actuelle? Quels sont les chercheurs Européens qui s'intéressent à l'éducation des communautés marginalisées en Europe?

E: Tu as raison, c'est une question d'actualité. Certaines études suggèrent en effet des différences dans la façon dont les enseignements de mathématiques sont assimilés par des enfants de différentes cultures (PISA notamment). Néanmoins, il s'agit là d'une question délicate; il faudrait peut-être commencer par s'interroger sur les méthodologies de recherche qui ont menées à de tels résultats. Parmi les travaux ethnomathématiques consacrés aux pratiques mathématiques de minorités autochtones européennes, j'ai trouvé intéressant le travail de Charoula Stathopoulou (2006) consacré à l'éducation mathématique à destination d'enfants appartenant à des communauté Rom. En s'intéressant à la langue vernaculaire, et en étudiant les systèmes de numérations développés dans cette langue, elle a cherché à comprendre - au travers une étude de terrain - les obstacles aux apprentissages mathématiques de ces enfants; obstacles didactiques que doivent surmonter leurs enseignants.

La prise en compte (voire l'apprentissage) des langues vernaculaires est essentiel pour mieux saisir les concepts (ethno-)mathématiques développés dans des sociétés autochtones. Dans le cadre 
du projet ETKnoS (mentionné plus haut) que je coordonne, nous collaborons avec des linguistes pour mener une analyse sémantique des termes techniques - utilisés par les praticiens de jeux de ficelle -, et expliciter les différents usages de ces termes, dont certains semblent être spécifiques à la pratique étudiée, tandis que d'autres sont utilisés dans plusieurs champs d'activités.

MC: Ici au Brésil, des chercheurs comme mon jeune collègue Kécio Leite, qui travaille avec des populations autochtones, s'intéresse effectivement aux langues vernaculaires, et aux difficultés de traduction de certains concepts mathématiques; traduction qui pose souvent problème dans les deux sens.

\section{Défis pour l'ethnomathématique aujourd'hui}

MC: Personnellement, je m'intéresse de plus en plus au "développement de l'ethnomathématique", envisagé comme un domaine de recherche à part entière. Cela nous ramène à notre collaboration. J'ai commencé par l'ethnomathématique brésilienne, puis j'ai constitué un réseau international (en participant à des colloques internationaux consacrés à l'éducation et/ou l'ethnomathématique). J'ai pu ainsi percevoir des différences d'approche en ethnomathématique dans les différentes régions du monde.

Récemment, il y a eu des évènements importants pour l'ethnomathématique qui se sont tenus en Amérique du sud et Amérique centrale ${ }^{8}$. Enfin, la constitution par Hilbert Blanco-Alvarez du réseau "Red latinoamericana de etnomatematica" (RELAET), devenu le "Red International de Etnomatemática” en 2019, a été important pour le domaine.

Les différentes approches de l'ethnomathématique sont directement liées à l'histoire des différents pays ou régions dans lesquels ce champ de recherche se développe, et aux études que les premiers chercheurs de ces pays ont réalisées. Au Brésil, l'influence de D’Ambrosio est étonnante. De fait, il est difficile de trouver une production brésilienne qui ne cite pas D’Ambrosio.

Nous l'avons déjà évoqué, le Brésil a été pionnier dans ce champ de recherche, dont D'Ambrosio a été le principal diffuseur. La question que je me pose maintenant, est de mieux comprendre comment ces recherches ont circulé dans des pays dont la langue n'est pas le portugais, et comment cette production brésilienne est perçue au-delà du Brésil. Toi-même, tu me disais que le portugais avait été une barrière. Mon nouveau projet ${ }^{9}$ vise donc à analyser la production européenne

\footnotetext{
${ }^{8}$ En 2018, le Sixième Congrès International en Ethnomathématique (ICEm6), s'est passé en Colombie; en 2019 Costa Rica a accueilli le Segundo Encuentro Latinoamericano de Etnomatemática (ELEm2).

${ }^{9}$ Projet intitulé "Marcas da produção acadêmica brasileira na pesquisa etnomatemática europeia", soutenu par une bourse d'initiation scientifique de CNPq (2020-2021).
} 
afin de mieux saisir les différentes conceptions de l'éthnomathématique, et les éventuelles influences du Sud vers le Nord.

Ce qui nous amène aux études sur la « decolonialité », qui m’ont intéressé plus récemment, et que l'on étudie, mon collègue Adriano Vargas Freitas et moi-même, avec nos groupes de recherche respectifs. Il faudrait un vrai "mouvement décolonisateur de l'ethnomathématique" (BERNALES; POWELL, 2018). Ici au Brésil, on parle beaucoup de ces questions en ce moment, surtout en raison des mouvements menés par des populations ayant des racines africaines et autochtones, cherchant une réparation historique des violences et du racisme subis depuis l'époque coloniale jusqu'à nos jours.

E: Ton nouveau projet de recherche, visant à mieux saisir la façon dont se développe l'ethnomathématique d'un pays/continent à l'autre permettra à terme d'avoir une vision plus précise de ce champ interdisciplinaire tel qu'il est constitué aujourd'hui. Mieux comprendre ce qui est en train de se passer - en prenant de la hauteur - est, me semble-t-il, un défi important pour l'ethnomathématique.

De mon côté, si je m'enrichis de nos discussions collectives sur ce thème, ainsi que sur celui de l'usage des savoirs traditionnels dans l'enseignement des mathématiques à destination des peuples autochtones, cela ne constitue pas, comme tu le sais, le cœur de mon travail. Mon projet vise plus particulièrement à étudier des activités à caractère mathématique (jeux de ficelle, dessins sur le sable, confection de nattes...), pratiquées dans des sociétés où prédomine l'oralité, et dans leurs liens avec des systèmes culturels spécifiques. Il me semble qu'il s'agit là également d'un défi pour l'ethnomathématique aujourd'hui.

De fait, si les ethnomathématiciens s'intéressent depuis longtemps aux activités techniques que l'on peut identifier comme relevant d'une pratique mathématique, nous manquons cruellement d'études approfondies consacrées à ces pratiques à caractère algorithmique et géométrique, comme celle des jeux de ficelle à laquelle j'ai consacré une thèse, plusieurs articles et un livre (2015c). J'entends contribuer à des avancées dans ce domaine en coordonnant des projets collectifs financés (comme le projet ETKnoS en cours) qui permettent à des chercheurs de mener des travaux sur ces pratiques (dessins sur le sable, production textile, tatouages...), en s'appuyant sur un travail ethnographique pour étudier ces activités in situ dans différentes sociétés. Certes, des travaux consacrés à certaines de ces activités ont été menés par l'anthropologie des techniques, en France dès les années 1970 notamment. Néanmoins, si ces travaux s'attachent le plus souvent à décrire ces techniques par le menu, ils ne donnent pas toujours les informations utiles à l'éthnomathématicien. Il est essentiel de mener des recherches ethnographiques dans une perspective ethnomathématique, pour 
comprendre la logique de ces activités, mieux saisir les processus cognitifs impliqués, et la nature des relations entre ces pratiques et les mathématiques.

MC: Je pense que les travaux consacrés aux études décoloniales (LANDER, 2000) offrent des concepts qui devraient nous permettre de mieux analyser les données collectées par les ethnomathématiciens sur le terrain. Ces derniers gagneraient probablement à ne plus mener des recherches sur des groupes sociaux, mais plutôt avec eux (SANTOS, 2019). À cet égard, je trouve intéressant le concept d' "interrogation mutuelle”, introduit par Willy Alangui (2010). Il fait le constat que les rencontres entre différents différents groupes socioculturels sont inévitables et continues, et que ce phénomène implique des transformations culturelles. La mission de l'ethnomathématicien serait alors de faciliter/organiser le dialogue critique entre les mathématiques et d'autres systèmes de connaissance dans diverses sociétés.

L'ethnomathématique comme domaine interdisciplinaire est donc confronté à nombre de défis dont certains sont au cœur de notre collaboration.

E: Oui, je suis d'accord, et j'espère que notre conversation permettra à nos lecteurs d'en percevoir les enjeux.

\section{Références}

\section{ALANGUI, Wilfredo V. Stone Walls and Water Flows: Interrogating Mathematics and} Cultural Practice. Auckland: The University of Auckland, 2010.

ASCHER, Marcia. Ethnomathematics: A multicultural view of mathematical ideas. Pacific Grove, California: Brooks and Cole Publishing Company, 1991.

ASCHER, Marcia. Mathématiques d'ailleurs : nombres, formes et jeux dans les sociétés traditionnelles. Paris: Editions du Seuil, 1998 (Traduction par Karine Chemla et Serge Pahaut de Ethnomathematics: A multicultural view of mathematical ideas).

ASCHER, Marcia; D'AMBROSIO, Ubiratan. Ethnomathematics: a Dialogue. For the Learning of Mathematics, 14(2), p. 36-43, 1994.

BERNALES, Martha; POWELL, Arthur B. Decolonizing Ethnomathematics. Ensino Em-Revista v. 25, n.3, p. 565-587, set/dez 2018.

BULF, Caroline. Le rôle de la symétrie dans la nature du travail géométrique des tailleurs de pierre et ébénistes. Annales de sciences cognitives, 15, p. 117-139, 2010.

CAMPOS, Marcio D’Olne. Etnociência ou Etnografia de Saberes, Técnicas e Práticas? Anais do I Seminário de Etnobiologia e de Etnoecologia do Sudeste. Rio Claro, 2001.

CARRAHER, Terezinha, CARRAHER, David; SCHLIEMANN, Annalúcia. Na vida dez, na escola zero. São Paulo: Cortez, 1989. 
CASTELA, Corine, \& ELGUERO, Cecilia. Praxéologie et institution, concepts clés pour l'anthropologie épistémologique et la socioépistémologie. Recherches En Didactique Des Mathématiques, 33(2), p. 123-162, 2013.

CHEMILLIER, Marc. Les Mathématiques naturelles. Paris: Odile Jacob, 2007.

CHEMLA, Karine (Ed.). The History of Mathematical Proof in Ancient Traditions. Cambridge: Cambridge University Press, 2012

D’AMBROSIO, Ubiratan. Etnomatemática. São Paulo: Editora Ática, 1990.

D'AMBROSIO, Ubiratan. Etnomatemática: Elo entre as tradições e a modernidade. Belo Horizonte: Autêntica, 2001.

DE VARGAS, Sonia. .Estratégias não-escolares de ensino-aprendizagem e formação de professores da EJA. In: FANTINATO, Maria Cecilia (org.) Etnomatemática: novos desafios teóricos e pedagógicos (pp.193-201). Niterói: Editora da UFF, 2009.

FANTINATO, Maria Cecilia. A construção de saberes matemáticos entre jovens e adultos do Morro de São Carlos. Revista Brasileira de Educação, v.27, p.109-124, set/dez 2004.

FANTINATO, Maria Cecilia (org.) Etnomatemática: novos desafios teóricos e pedagógicos. Niterói: Editora da UFF, 2009.

FANTINATO, Maria Cecilia. Balanço da produção acadêmica dos congressos brasileiros de Etnomatemática. Revista Iberoamericana de educación matemática, v.1, n. 33, p.147-161, mar. 2013.

FANTINATO, Maria Cecilia; LEITE, Kécio Gonçalves. Ethnomathematics Research on Indigenous Peoples' Knowledge and Practices In: ROSA, Milton; OLIVEIRA, Cristiane Coppe (eds.) Ethnomathematics in Action. Cham: Springer International Publishing, p. 111-137, 2020.

FORMIGOSA, Marcos Marques; LUCENA, Isabel Cristina Rodrigues; SILVA, Carlos Aldemir Farias. Um navegar pelos saberes da tradição na Amazônia ribeirinha por meio da Etnomatemática. Revista Latinoamericana de Etnomatemática, v. 10, n. 1, p.1-14, 2017.

FRANÇOIS, Karen; SOUZA MAFRA, José R.; FANTINATO, Maria-Cecilia; VANDENDRIESSCHE, Eric. Local Mathematics Education: The implementation of local mathematical practices into the mathematics curriculum. Philosophy of Mathematics Education Journal, 33, p. 1-18, 2018.

LAMBELET, Alexandre; PETIT, Céline; VANDENDRIESSCHE, Eric; WENDLING, Thierry. Ethnologie et mathématiques. ethnographiques.org, 29, 2015 [en ligne] (https://www.ethnographiques.org/2014/numero-29/ - consulté le 09.09.2020)

LANDER, Edgardo (org.) La colonialidad del saber: eurocentrismo y ciencias sociales. Perspectivas latinoamericanas. Buenos Aires: Clacso, 2000.

MAFRA, José Ricardo; FANTINATO, Maria Cecilia. Artesãs de Aritapera/PA: técnicas e processos em uma perspetiva Etnomatemática. Revista Latinoamericana de Etnomatemática, v. 9, n.2, p. 180-201, jun/set 2016. 
MARCHON, Fabio Lennon; FANTINATO, Maria Cecilia. Possibilidades filosóficas em Etnomatemática. Bolema, v. 29, n. 52, p. 549-567, ago. 2015.

MARCHON, Fabio Lennon; FANTINATO, Maria Cecilia. Aspectos poéticos e retóricos na composição do enredo da narrativa histórica da Etnomatemática. Zetetiké, v.27, p.1-14, 2019.

MEIRA, Claudia de Jesus; FANTINATO, Maria Cecilia. Os saberes matemáticos de jovens e adultos em contexto de privação de liberdade. Revista Latinoamericana de Etnomatemática, v.8, p.177 - 193, jun/set 2015 .

MONTEIRO, Alexandrina; MENDES, Jackeline Rodrigues. A etnomatemática no encontro entre práticas e saberes: convergências, tensões e negociação de sentidos. Revista Latinoamericana de Etnomatemática V.7, n. 3, p.55-70, out 2014.

OLIVEIRA, Maria Aparecida Mendes; MENDES, Jackeline Rodrigues. Formação de professores Guarani Kaiowá: interculturalidade e decolonialidade no ensino de matemática. Zetetiké, v.26, n.1, p. 167-184.jan/abr 2018.

PAIS, Alexandre. Criticisms and contradictions of ethnomathematics. Educational Studies in Mathematics, 76 (2), p. 209-230, 2011.

PASSOS, Caroline Mendes. Condições de Produção e Legitimação da Etnomatemática. Tese (doutorado). Programa de Pós-Graduação em Educação da Universidade Federal de São Carlos: São Carlos, SP. 2017.

RADFORD, Luis. L'ethnomathématique au carrefour de la recolonisation et la décolonisation des savoirs. In: MAHEUX, G.; QUINTRIQUEO, S.; PELLERIN, L.; BACON, L. (Eds.), La décolonisation de la scolarisation des jeunes inuit et des Première Nations : sens et défis (pp. 247-276). Montréal: Presses de l’Université du Québec, 2020.

ROSA, Milton; OLIVEIRA, Cristiane Coppe (orgs.) Ethnomathematics in Action. Cham: Springer International Publishing, 2020.

SANTOS, Boaventura de Souza. O fim do império cognitivo: a afirmação das epistemologias do Sul. Belo Horizonte: Autêntica, 2019.

SILVA, Vanísio Luiz; COSTA, Wanderleya Nara Gonçalves. Matemática, corpo e resistência cultural. Ensino Em Re-Vista, v. 25, n.3, p. 609-629, set/dez. 2018.

STATHOPOULOU, Charoula. Cultural and Cognitive Conflicts in School Context: Mathematics Education as an Acculturation Process for Romany Students. Themes in Education, 7 (2), p. 131143, 2006.

SURUÍ, Adriano Pawah; LEITE, Kécio Gonçalves. Etnomatemática e Educação Escolar Indígena no contexto do povo Paiter. Zetetiké, v.26, n.1, p.94-112, jan/abr 2018.

VANDENDRIESSCHE, Eric. Kaninikula, mathématiques aux îles Trobriand. Documentaire scientifique, 29 min, Production CNRS, diffusion CNRS Images, 2012. URL:

http://videotheque.cnrs.fr/doc $=4149$ ?langue $=$ FR 
VANDENDRIESSCHE, Eric. De l'ethnomusicologie à l'ethnomathématique. Entretien avec Marc Chemillier. In: LAMBELET, Alexandre; PETIT, Céline; VANDENDRIESSCHE, Eric; WENDLING, Thierry. Ethnologie et mathématiques. ethnographiques.org, 29 (déc. 2014), 2015(a). https://www.ethnographiques.org/2014/Vandendriessche-Chemillier (consulté le 09.09.2020)

VANDENDRIESSCHE, Eric. Ethnomathématique des jeux de ficelle trobriandais. In:

LAMBELET, Alexandre; PETIT, Céline; VANDENDRIESSCHE, Eric; WENDLING, Thierry. Ethnologie et mathématiques. ethnographiques.org, 29 (déc. 2014), 2015(b) https://www.ethnographiques.org/ethnomathematique-des-jeux-de-ficelle-trobriandais (consulté le 09.09.2020)

VANDENDRIESSCHE, Eric. String figures as mathematics? An Anthropological Approach to String Figure-making in Oral Tradition Societies. Studies in History and Philosophy of Science 36. Cham: Springer, 392p, 2015(c).

VANDENDRIESSCHE, Eric; SOUZA MAFRA, José R.; FANTINATO, Maria-Cecilia; FRANÇOIS, Karen. How Local Are Local People? Beyond Exoticism. In A. Chronaki (ed.) Mathematics education and life at times of crisis. Proceedings of the Ninth International Mathematics Education and Society Conference-MES- Vol 2. (pp. 956-967). Book Series: Mathematics Education and Society. Volos, Greece, April 7-12, 2017.

VANDENDRIESSCHE, Eric; PETIT, Céline. Des prémices d'une anthropologie des pratiques mathématiques à la constitution d'un nouveau champ disciplinaire : l'ethnomathématique. Revue d'histoire des sciences humaines, 31, p. 189-219, 2017. 